\title{
Side Band Power reduction in OFDM
}

\author{
S.P.Mohani \\ Assistant Professor \\ Dept. of Electronics \& Telecom \\ College of Engg, Pune
}

\author{
Dr. M.S. Sutaone \\ Professor \\ Dept. of Electronics \& Telecom \\ College of Engg, Pune, India
}

\author{
Meeta Bakuli \\ Sr. Lecturer \\ Dept. Of Electronics \\ GHRCEM,Pune,India
}

\begin{abstract}
In the recent past, wireless communications have experienced a fast growth due to the high mobility that they allow. However, wireless channels have some disadvantages, like multi-path fading, that make them difficult to deal with. A modulation scheme that efficiently deals with selective fading channels is OFDM. An important disadvantage of OFDM systems is their high peak-toaverage power ratio (PAPR). Clipping and filtering can be a simple and effective method of reducing the peak-to-average power ratio (PAPR). But this results in an increase in Side Band / out-of-band (OOB) power, which increases PAPR. In this paper, it is shown that if the OOB power is attenuated in step by step fashion instead of abrupt reduction, then PAPR can also be reduced. Simulation results related to this using MATLAB are presented.
\end{abstract}

\section{INTRODUCTION}

Orthogonal Frequency Division Multiplexing (OFDM) technology is used in many broadband communications systems due to its robustness against frequency selective fading and impulse noise, high bandwidth efficiency and relatively simple receiver implementation. One of the main disadvantages of OFDM is its high peak-to-average power ratio (PAPR). OFDM transmitters therefore require highly linear output amplifiers with wide dynamic range. Such amplifiers are inefficient, expensive and can cause excessive drain on the batteries of portable equipment in applications such as wireless local area networks (WLAN),. Any amplifier nonlinearity causes inter-modulation products resulting in unwanted side band / out-of-band (OOB) power. The PAPR of an OFDM signal can be reduced in several ways. One of the techniques is Selective mapping [1,2], which involves generating a large set of data vectors all representing the same information. The data vector with the lowest resulting PAPR is selected. Information regarding the particular selected data vector is sent as additional side information to the receiver. However, in selective mapping, there may be potential problems with decoding the signal in the presence of noise. Reverse mapping would result in the loss of data of whole symbols.

The simplest approach to reduce the PAPR in OFDM signals is to clip the high amplitude signals. Clipping results in out-of-band (OOB) power and some in-band distortion, but it has been shown that, in practice, the effect of this on overall bit error rate (BER) is negligible [9]. This is because the main effect of clipping is to shrink the overall signal constellation rather than to add clipping noise. Moreover, the clipping noise is added at the transmitter, rather than the receiver and is subject to frequency selective channel fading along with the wanted component signals.

In this paper, it is shown that along with the reduction in PAPR, the amount of the OOB power that is allowed at the output of the filter is reduced. Simulation results in which some OOB power is allowed at the output of the filter are shown. The amount of OOB power is set according to the frequency offset of the sub-carriers. Limiting the OOB power of the FFT based filter causes less peak re-growth than the linear filter.

\section{BACKGROUND}

In OFDM, a block of $\mathrm{N}$ symbols, $\{\mathrm{Xn}, \mathrm{n} \square \square 0,1, \ldots,(\mathrm{N}-1)\}$, is formed with each symbol modulation, one of a set of $\mathrm{N}$ subcarriers, $\{$ fn , $\mathrm{n} \square \square, 1, \ldots, \mathrm{N} \square 1\}$. The $\mathrm{N}$ sub-carriers are chosen to be orthogonal, that is, $\square$ fn $\square \square \mathrm{n} \Delta \mathrm{f}$, where $\Delta \mathrm{f} \square 1 / \mathrm{NT}$ and $\mathrm{T}$ is the original symbol period. The resulting signal after digital to analog conversion can be expressed as

$$
x(t)=\sum_{n=0}^{N-1} x n \text { ej2 } 2 \pi n t \quad 0 \leq t \leq N T s
$$

These time-domain samples in the equivalent complex valued lowpass domain are approximately Gaussian distributed because of the statistical independence of carriers.

The resulting high PAR is given by

$$
\text { PAPR }=\max _{E\left[\left.\left.|x(t)|\right|^{2}\right|^{2}\right.}
$$

Fig. 1 shows serial to parallel conversion of input bit-stream and imposition of these parallel bit-streams on orthogonal sub-carriers and the resultant OFDM signal.

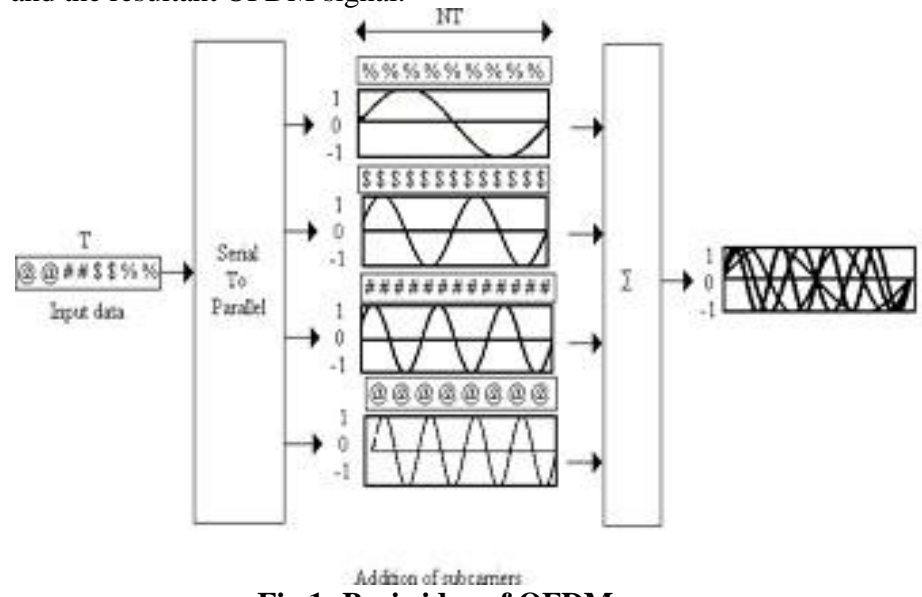

Fig.1: Basic idea of OFDM

If the number of sub-carriers is large enough, magnitudes of real and imaginary part of output signal $\mathrm{s}(\mathrm{t})$ have Gaussian distribution, and the amplitude of the OFDM signal follows Rayleigh distribution. Power distribution of OFDM signal is central chisquare distribution with 2 degree of freedom and a mean of zero The probability density function (PDF) of power is 


$$
F(z)=1-e^{-z}
$$

where $z$ is special threshold. Assume that sampled values of PDF in maximum power per OFDM symbol are uncorrelated. In the case of non-oversampling, CCDF, the probability that PAPR is smaller than threshold, is

$$
C C D F=P(P A P R \leq z)=F(z)^{N=}\left(1-e^{-z}\right)^{N}
$$

The assumption made in deriving (4) that the samples should be mutually uncorrelated is no longer valid when over-sampling is applied. The effect of over-sampling is approximated by adding a certain number of extra independent samples. The distribution of the PAPR is then given by

$$
P(P A P R \leq z)=\left(1-e^{-z}\right)^{\alpha N}
$$

\section{OUT-OF-BAND POWER IN OFDM}

The spectrum of the OFDM signal has some OOB power. The form of the OOB spectrum depends very strongly on the detailed design of the OFDM transmitter. However, the OOB power is mainly due to two factors: the sidelobes of the spectra of the in-band subcarriers and intermodulation products due to nonlinearities in the transmitter output. In the case of an OFDM system with no windowing of each output symbol, each subcarrier has a $\sin (x) / x$ spectrum. This results in relatively high side-lobes. The nonlinearities are due to non-ideal characteristics of the amplifier, or due to deliberate clipping of the signal to eliminate signal peaks. It has been shown that the limiting factor becomes the amplifier nonlinearity when clipping is used [8]. The clipping function is performed in digital time domain and is described by the expression:

$$
\begin{aligned}
x_{c}{ }^{k} & =x^{k} & \left|x_{k}\right| \leq A & , 0 \leq k \leq N-1 \\
& =A e^{j \phi(x k)} & \left|x_{k}\right|<A & , 0 \leq k \leq N-1
\end{aligned}
$$

where $x_{k}^{c}$ is the clipped signal, $x_{k}$ is the transmitted signal, $A$ is the clipping amplitude and $\varphi\left(x_{k}\right)$ is the phase of $x_{k}$. The graphical expression of this function is shown in Figure 2.

The clipping ratio $(\mathrm{CR})$ is defined as the ratio of the clipping level to the root-mean-square power of the unclipped baseband signal,

$$
C R=\frac{A}{O}
$$

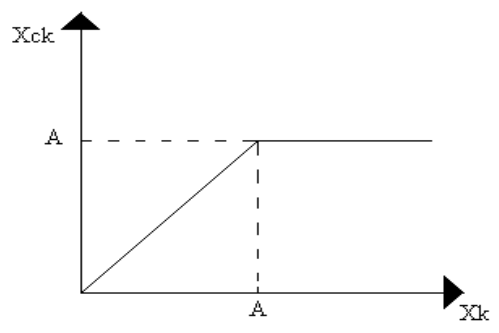

Fig 2: Clipping function

$$
C R[d B]=20 \log \underline{A}
$$

where ó is the root mean squared value of the unclipped OFDM signal and its mathematical expression is

$$
o^{2}=\sum_{k=1}^{N} x_{k}^{2}
$$

Clipping, being a non-linear process, introduces in-band distortion also called clipping noise, out-of-band radiation and inter-carrier interference (Fig. 3), which degrades the system performance and the spectral efficiency.

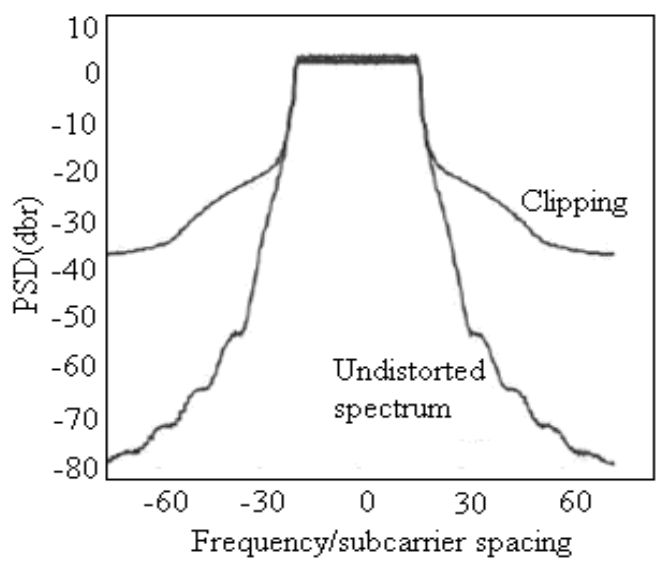

Fig 3: Out of Band Radiation

The clipping noise is related to the difference between the original signal $x_{k}$ and the clipped signal $x_{k}^{c}$. The signal sent to the receiver is the clipped signal, which is obviously different from the original signal. This difference is measured by signal to clipping noise ratio (SCNR), given by:

$$
\text { SCNR }=\frac{\left|x_{c}{ }^{k}\right|^{2}}{\left|x_{k}-x_{c}\right|^{2}}
$$

The more the signal is clipped, the greater is the difference between clipped and unclipped signals and the smaller will be the SCNR. On the other hand, the less the signal is clipped, the smaller is the difference between clipped and unclipped signals and higher will be the SCNR.

\section{TECHNIQUE FOR OOB POWER REDUCTION}

The clipping ratio in $\mathrm{dB}$ is given by 


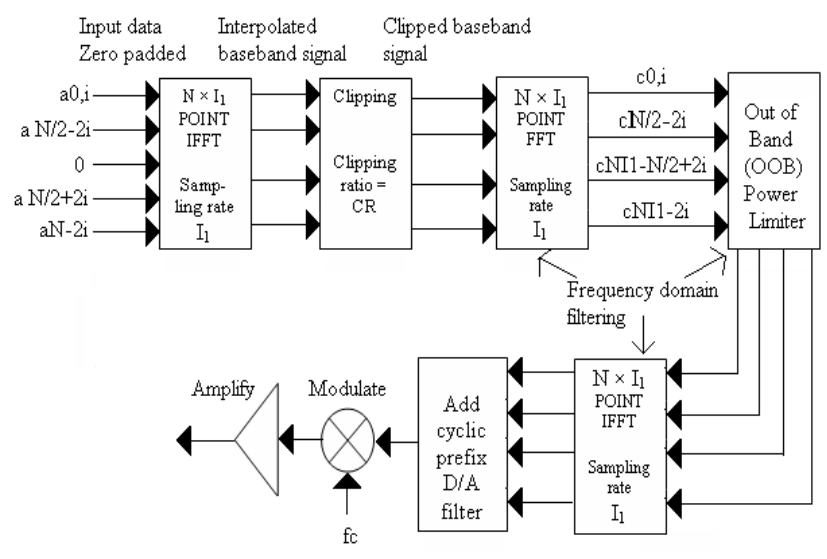

Fig 4: Block Diagram of OOB Power reduced OFDM system

Fig. 4 shows the block diagram of the new FFT based OOB reduction scheme. Vector $A_{i}=a_{0}, a_{1} \ldots, a_{N-1}$, which represents the data in each symbol $i$, is converted from frequency to time domain using an oversized IFFT. $\mathrm{N}$ is the number of subcarriers in each OFDM symbol. For an oversampling factor of $I_{1}$, the input vector is extended by adding $\mathrm{N}\left(\mathrm{I}_{1}-1\right)$ zeros in the middle of the vector. That is,

$i=\left[a_{0, i}, a_{1, i}, \quad 0, \ldots \ldots, 0 \quad \ldots \ldots a_{N-1, i}\right]$

This results in trigonometric interpolation of the OFDM time domain signal .The discrete time domain OFDM signal oversampled by a factor of $\mathrm{I}_{1}$ can be expressed as,

$$
x(n)=\sum_{k=0}^{N-1} a(k) \exp \left(j 2 \pi n k / N I_{1}\right)
$$

The interpolated signal is then clipped using amplitude clipping with various clipping ratios. Clipping is followed by frequency domain filtering to limit the OOB power. The filter consists of a FFT followed by an IFFT operation. The clipped signal $\mathrm{c}_{0}, \ldots$, $\mathrm{c}_{\mathrm{N} / 2-1}, \mathrm{c}_{\mathrm{NI1}-\mathrm{N} / 2+1}, \ldots, \mathrm{c}_{\mathrm{NI1}-1}$ are passed unchanged while OOB power spectral components are modified according to the OOB block specification. This is different from the FFT filter described in [6] where all of the OOB components are nullified rather than limited.

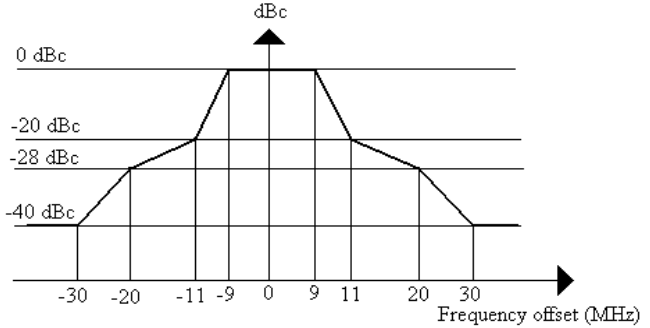

Fig 5: OOB reduction of Subcarriers

Fig. 5 shows that each OFDM symbol is composed of 64 subcarriers with a nominal bandwidth of $20 \mathrm{MHz}$. Out of the 64 subcarriers, 47 are data carriers, 4 are pilots. The remaining 13 subcarriers (the $0^{\text {th }}$ and some band-edge subcarriers) are not used and are set to zero. The band-edge subcarriers are not used as this reduces the analog filtering requirements. The information bearing subcarriers have an effective bandwidth of $18 \mathrm{MHz}$ while OOB power decays below $40 \mathrm{dBc}$ at a frequency offset of $30 \mathrm{MHz}$ away from the carrier frequency.

\section{PERFORMANCE OF PAPR REDUCTION}

Simulation Results for this algorithm using MATLAB are shown. Fig. 7 and 8 show the complementary cumulative distribution function (CCDF) of PAPR for CR values of 4.5 and $2.5 \mathrm{~dB}$ respectively. Clearly if OOB masking is applied, it can be observed that negligible peak power re-growth occurs.

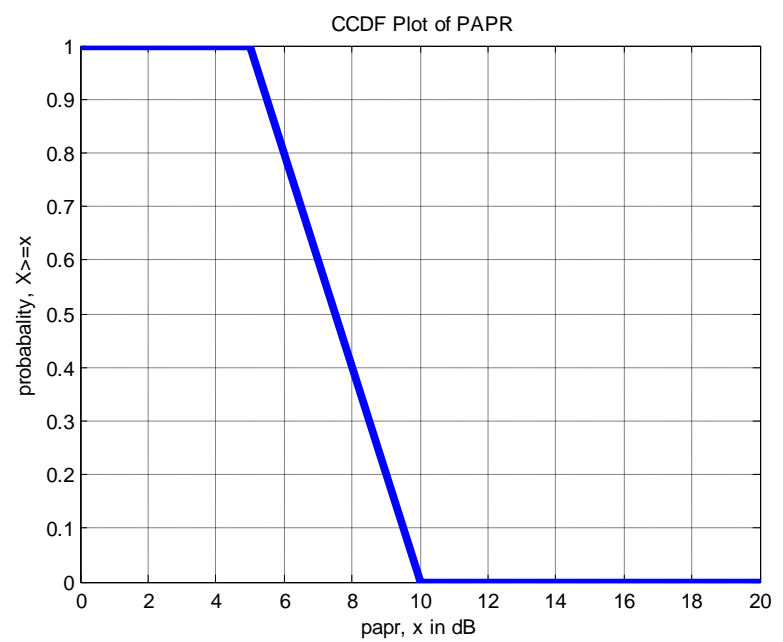

Fig 6: CCDF of PAPR (Block 1) for Original Signal

Fig 6 shows the CCDF plot of Block 1 for Original OFDM Signal. 


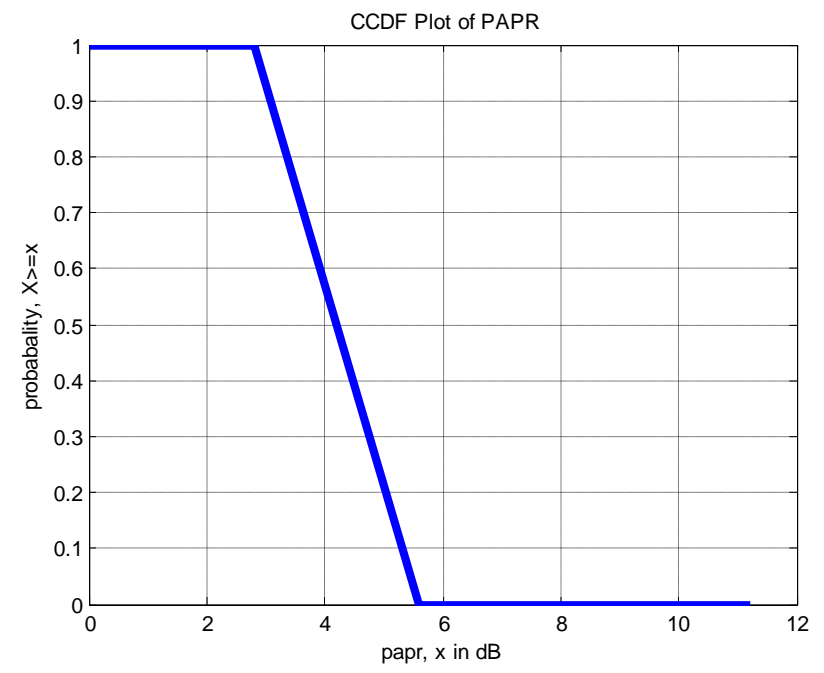

Fig 7: CCDF of PAPR (Block 2) for $\mathrm{CR}=2.5 \mathrm{~dB}$

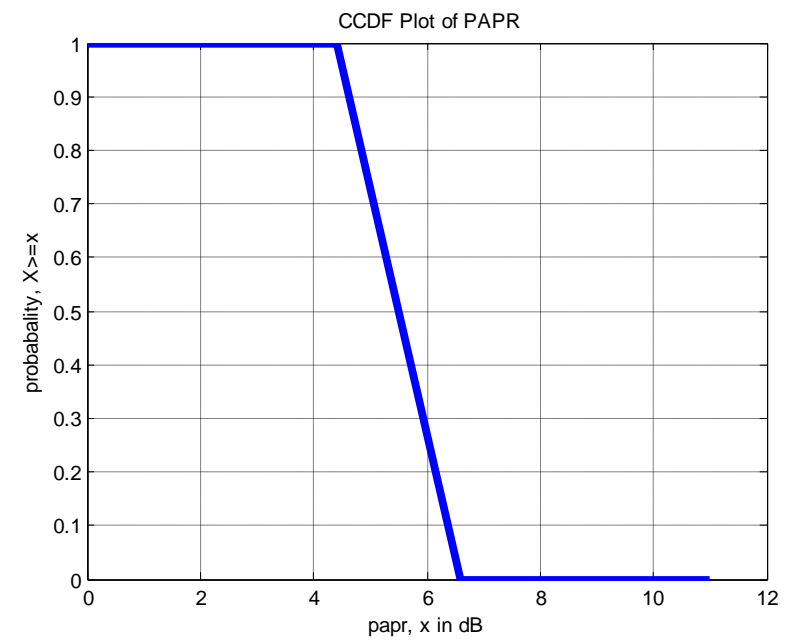

Fig 8: CCDF of PAPR(Block 2) for $C R=4.5 \mathrm{~dB}$

Fig 7 shows the CCDF plot of Block 2(Clipping Block) of fig 4 for a $\mathrm{CR}=2.5 \mathrm{~dB}$.

Fig 8 shows the CCDF plot of Block 2 (Clipping Block) of fig 4 for a $\mathrm{CR}=4.5 \mathrm{~dB}$. It clearly shows that for $\mathrm{CR}=4.5 \mathrm{~dB}$, PAPR is more than for $\mathrm{CR}=2.5 \mathrm{~dB}$.

Fig 9 shows the Spectral Power mask of OFDM.

Fig 10 shows the CCDF plot of Block 5(OOB Power Block) of fig 4 for $\mathrm{a} \mathrm{CR}=2.5 \mathrm{~dB}$.

Fig 11 shows the CCDF plot of Block 5 (OOB Power Block) of fig 4 for $\mathrm{a} \mathrm{CR}=4.5 \mathrm{~dB}$.

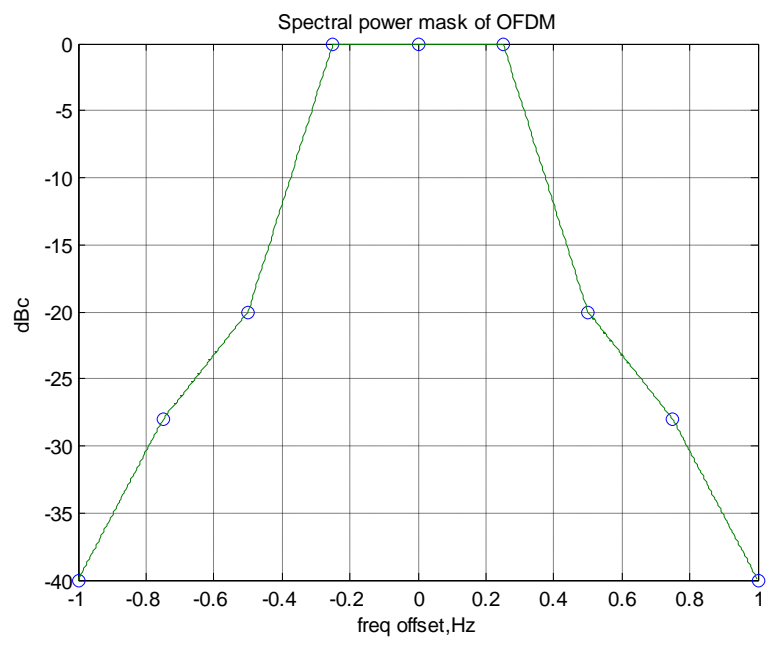

Fig 9: Spectral Power Mask Of OFDM.

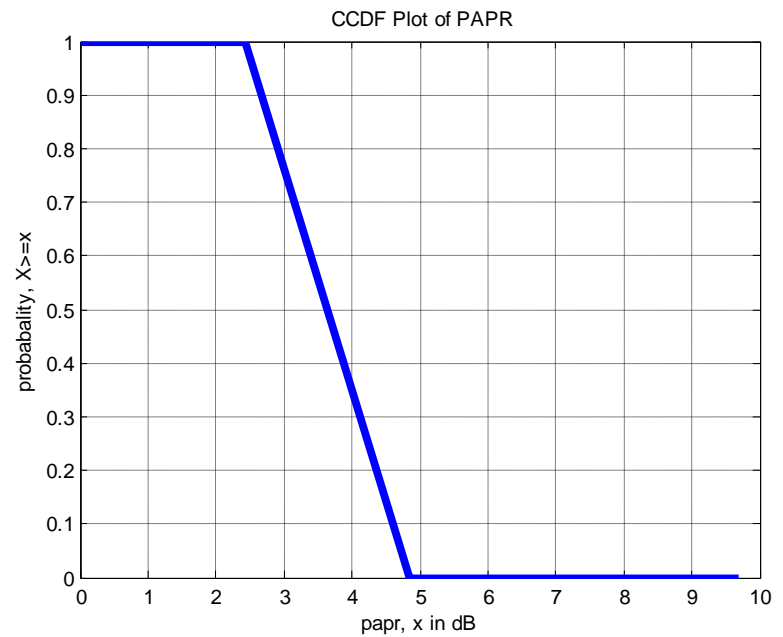

Fig 10: CCDF of PAPR (Block 5)for CR $=2.5 \mathrm{~dB}$

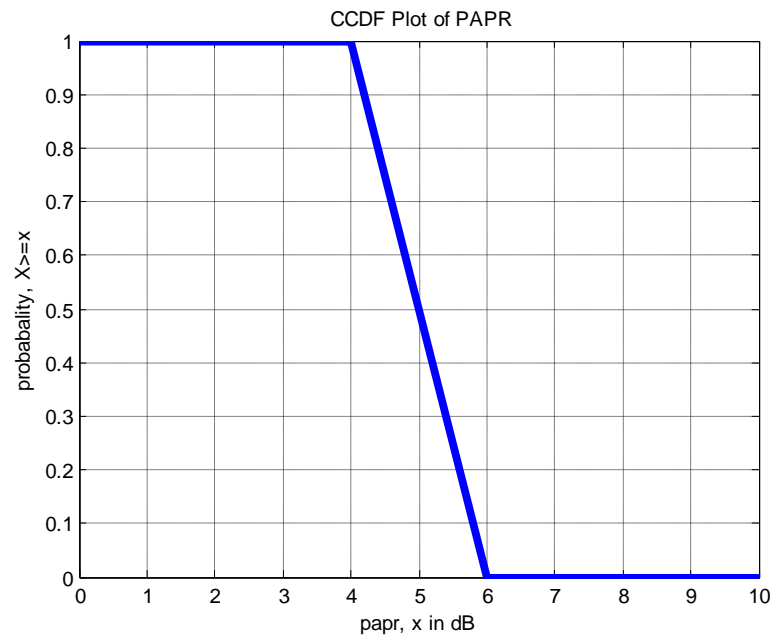

Fig 11: CCDF of PAPR (Block 5)for $C R=4.5 \mathrm{~dB}$ 


\section{CONCLUSION}

A detailed analysis of a FFT based filter to reduce the peak-toaverage power ratio (PAPR) in OFDM signals is presented. Side Band / OOB Power Block is used to limit the out-of-band (OOB) power and it is shown that the resulting peak-regrowth will be minimum. FFT based OOB Power filter introduces no in-band distortion. The effect of various values of clipping ratio on the PAPR of OFDM system is studied in detail.

\section{REFERENCES}

[1] Hara, S. Prasad, R. "overview of multicarrier CDMA", IEEE Communications Magazine, Vol. 35, No. 12, pp126133,December, 1997.

[2] Xiaodong Li and Leonard J. Cimini, Jr., "Effect of Clipping and Filtering on the Performance of OFDM"

[3] X. Li and L. J. Cimini Jr., "Effects of clipping and filtering on the performance of OFDM", IEEE Commun. Lett., vol. 2, pp. 131-133, May 1998.

[4] R. O’Neill and L. N. Lopes, "Envelope variations and spectral splatter in clipped multicarrier signals,“" in Proc. PIMRC '95, Toronto, Canada, Sept. 1995, pp. 71-75.

[5] C. Tellambura, "Phase optimization criterion for reducing peak-to-average power ratio for OFDM," IEE Electron. Lett., vol. 34, pp. 169-170, Jan. 1998.
[6] J. Armstrong, "Peak-to-average power reduction for OFDM by repeated clipping and frequency domain filtering", IEE Electron. Lett., vol. 38, pp. 246-247, Feb. 2002.

[7] J. Armstrong, "New OFDM peak-to-average power reduction scheme," in Proc. of IEEE Vehicular Technology Conf. (VTC' 2001 Spring), Rhodes, Greece, May 2001, pp. 756-760.

[8] L. D. Kabulepa, T. Pionteck, A. Garcia and M. Glesner, "Design exploration for clipping and filtering PAPR reduction techniques in OFDM systems", in Proc. 8 th International OFDM Workshop (InOWo'03), Hamburg, Germany, Sep. 2003, pp. 108-112.

[9] K. R. Panta and J. Armstrong, "Effects of clipping on the error performance of OFDM in frequency selective fading channels," IEEE Trans. Wireless Commun., vol. 3, pp. 668671, Mar. 2004.

[10] R. van Nee and R. Prasad, $O F D M$ for Multimedia Communications, Boston, MA: Artech House, 2000.

[11] D. Fraser, "Interpolation by the FFT revisited - an experimental investigation", IEEE Trans. Acoustics, Speech and Signal Processing, vol. 37, pp. 665-675, May 1989.

[12] ETSI TS 101 475: Broadband Radio Acess Networks (BRAN); HIPERLAN Type 2; physical (PHY) layer. 